\title{
Applications of GIS and Very High-Resolution RS Data for Urban Land Use Change Studies in Mongolia
}

\author{
D. Amarsaikhan, ${ }^{1,2}$ V. Battsengel, ${ }^{1}$ E. Egshiglen, ${ }^{1}$ R. Gantuya, ${ }^{1}$ and D. Enkhjargal1 \\ ${ }^{1}$ Institute of Informatics and RS, Mongolian Academy of Sciences, Avenue Enkhtaivan-54B, Ulaanbaatar-51, Mongolia \\ ${ }^{2}$ School of Geography and Geology, National University of Mongolia, Ikh Surguuliin gudamj-6, Ulaanbaatar-46, Mongolia
}

Correspondence should be addressed to D. Amarsaikhan, amar64@arvis.ac.mn

Received 13 July 2011; Accepted 22 November 2011

Academic Editor: Vito Pascazio

Copyright (C) 2011 D. Amarsaikhan et al. This is an open access article distributed under the Creative Commons Attribution License, which permits unrestricted use, distribution, and reproduction in any medium, provided the original work is properly cited.

\begin{abstract}
The aim of this study is to analyze the urban land use changes occurred in the central part of Ulaanbaatar, the capital city of Mongolia, from 1930 to 2008 with a 10-year interval using geographical information system (GIS) and very high-resolution remote sensing (RS) data sets. As data sources, a large-scale topographic map, panchromatic and multispectral Quickbird images, and TerraSAR synthetic aperture radar (SAR) data are used. The primary urban land use database is developed using the topographic map of the study area and historical data about buildings. To extract updated land use information from the RS images, Quickbird and TerraSAR images are fused. For the fusion, ordinary and special image fusion techniques are used and the results are compared. For the final land use change analysis and RS image processing, ArcGIS and Erdas imagine systems installed in a PC environment are used. Overall, the study demonstrates that within the last few decades the central part of Ulaanbaatar city is urbanized very rapidly and became very dense.
\end{abstract}

\section{Introduction}

At present, cities allover the world are experiencing rapid growth because of the rapid increase in the world population and the irreversible flow of people from rural to urban areas [1]. In the coming decades, the world's rapid urbanization will be one of the greatest challenges to ensure human welfare and global environment. According to recent estimates, cities occupy about four percent of the world's terrestrial surface, yet they are home to almost half of the global population, consume close to three-quarters of the overall natural resources, and generate three-quarters of its pollution and wastes. The UN estimates that virtually all net global population and economic growth over the next 30 years will occur in cities, leading to a doubling of current population [2].

In general, the developed countries have a higher percentage of urban people than the developing countries. However, rapid urbanization process is mainly occurring in less developed countries, and it is expected that in future most urban expansions will occur in developing countries.
Mongolia, as many countries of the developing world, has problems with the urban expansion and the growth of population in the main cities. For example, over the last two decades, Ulaanbaatar, the capital city of Mongolia, has experienced different urban related problems. In the city, various problems had been previously accumulated during the centralized economy, and they have been intensified by the reforms of the entire political and economic systems, unregulated market development, and the rapid population growth caused mainly by migration from rural areas $[1,3]$.

To prevent rapid urban expansion, urban planners and decision makers need to regularly evaluate development procedures using updated urban planning maps. However, many city planners in developing countries lack access to updated maps and often rely on old data that are not relevant [1]. One of the possible solutions could be the use of RS images with different spatial and spectral resolutions. As the present RS is so advanced, it is now possible to extract different thematic information at various scales, to integrate the extracted information with other historical data sets stored in a GIS and to conduct sophisticated analyses [1]. 
In recent years, imaging techniques have made tremendous progress. Although a static model of a Digital Earth is necessarily the starting point for any data analysis, we should consider more and more the importance of change as well as natural and man-made processes. In general, images contain a wealth of information, and they are utilised for many applications. Geoinformtion sciences increasingly make use of imaging technologies. Earth-observing satellite platforms carry increasingly very high-resolution imaging sensors [4]. Now the highest-spatial-resolution satellite images can be acquired with centimetres accuracy, whereas the ordinary high-resolution images can be acquired with a few metres accuracy [1]. This makes RS a very useful technique for Digital Earth applications and supporting tool for different planning and management, including urban planning.

It is clear that unplanned and uncontrolled urban expansion may have different negative consequences [5]. However, such kind of problem is rather new in Mongolia. In developed countries, it is common that the controlling and coordination of the land use types from a view point of urban planning are based on the research about urban internal structure and land use. However, in Mongolia, such research studies are rare. For example, in the case of Ulaanbaatar city, there is a lack of thorough studies in relation to the internal structure and land use types. Therefore, in order to investigate different problems of the capital city, it is vitally important to thoroughly investigate its fundamentals, that is the internal structure and land use types, and make a rapid decision in order to solve not only socioeconomic, but also the major urban environmental-ecological related problems [6].

The aim of this study is to analyze the urban land use changes occurred in the central part of Ulaanbaatar, the capital city of Mongolia, from 1930 to 2008 with a 10-year interval using RS and historical GIS data sets. For the basic preparation of spatial and attribute databases, a large-scale topographic map of 2000 and historical description of the buildings have been used. To update the database of 2000 up to the year of 2008, very high-resolution panchromatic and multispectral Quickbird images of 2006 and TerraSAR image of 2008 have been fused. For the fusion, different data fusion techniques have been compared in terms of the enhancement of spatial and spectral variations of urban features. To extract land use information from the fused images, a visual interpretation has been applied. The final analysis was carried out using ArcGIS 9.2 and Erdas Imagine 9.2 systems, and different techniques were applied.

\section{Test Site and Data Sources}

As a test site, Ikh Toiruu area of Ulaanbaatar, the capital city of Mongolia, has been selected. Although Ulaanbaatar is extended from the west to the east about $30 \mathrm{~km}$ and from the north to the south about $20 \mathrm{~km}$, the study area chosen for the present study covers the central part of the capital city (approximately $3.75 \mathrm{~km}$ long and $2.65 \mathrm{~km}$ wide) and has a ring structure. In the selected image frame, it is possible to define such main classes as building area, ger area (Mongolian traditional dwelling), road, bare soil, vegetation,

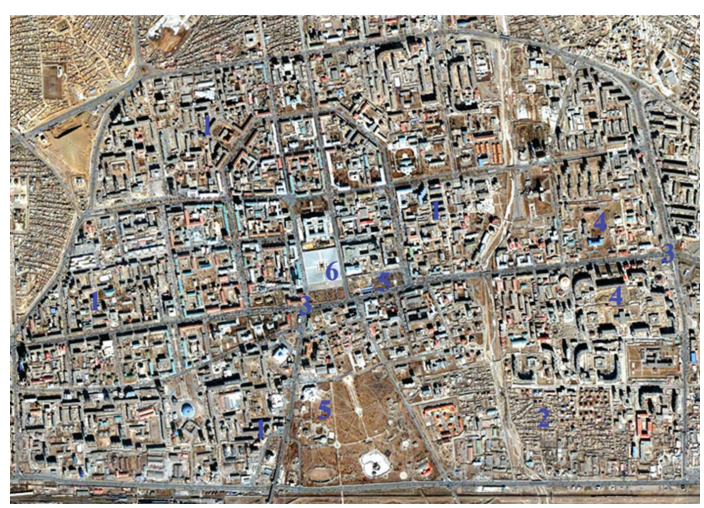

FIGURE 1: 2006 Quickbird image of the selected part of Ulaanbaatar. (1) Building area; (2) ger area; (3) roads; (4) bare soil; (5) vegetation; (6) central squire. The size of the displayed area is about $3.75 \mathrm{~km} \times 2.65 \mathrm{~km}$.

and central squire. Figure 1 shows a Quickbird image of the test site and some examples of its land cover.

In the present study, for the urban land use change study, 1:5000 scale topographic map of 2000, Quickbird images of March 2006, and VV polarization of TerraSARX image of March 2008 have been used. The Quickbird data has four multispectral bands (B1: $0.45-0.52 \mu \mathrm{m}, \mathrm{B} 2$ : $0.52-0.60 \mu \mathrm{m}, \mathrm{B} 3: 0.63-0.69 \mu \mathrm{m}$, and B4: $0.76-0.90 \mu \mathrm{m})$ and one panchromatic band (Pan: $0.45-0.9 \mu \mathrm{m}$ ). The spatial resolution is $0.61 \mathrm{~m}$ for the panchromatic image, while it is $2.4 \mathrm{~m}$ for the multispectral bands. In the current study, panchromatic, red, and near infrared bands have been used. TerraSAR-X is a German Earth observation satellite carrying a cloud-piercing, night-vision radar which is designed to create the most precise maps and images ever produced by a civilian space radar system. It images the Earth's surface at a rate of one million square $\mathrm{km}$ a day and provides information at various spatial resolutions. In this study, VV polarization of TerraSAR X-band (wavelength is $3.1 \mathrm{~cm}$ ) data with a spatial resolution of $1 \mathrm{~m}$ has been used.

\section{Coregistration of Panchromatic, Multispectral, and SAR Images}

In order to extract geometrically accurate thematic information, thorough georeferencing should be applied to the original RS images. Initially, the panchromatic Quickbird image has been georeferenced to a Gauss-Kruger map projection using a topographic map of 2000, scale 1:5000. The ground control points (GCPs) have been selected on well-defined cross-sections of roads, streets, and building corners and in total, 12 regularly distributed points were selected. For the transformation, a second-order transformation and nearestneighbour resampling approach [7] have been applied, and the related root mean square (RMS) error was 1.08 pixel. Likewise, the multispectral Quickbird image has been georeferenced to a Gauss-Kruger map projection using the same topographic map of the test area. For the transformation, the same number of GCPs has been used, and the related RMS 
error was 0.97 pixel. In each case of the georeferencing, an image was resampled to a pixel resolution of $1 \mathrm{~m}$.

Then, the TerraSAR VV polarization image was geometrically corrected, and its coordinates were transformed to the coordinates of the georeferenced Quickbird images. In order to correct the SAR image, 15 more regularly distributed GCPs were selected from different parts of the image. For the actual transformation, a second-order transformation was used. As a resampling technique, the nearest-neighbour resampling approach was applied and the related RMS error was 1.26 pixel. As both optical and microwave images had a very high spatial resolution, the errors of less than $1.4 \mathrm{~m}$ were considered as acceptable for further studies.

\section{Speckle Suppression of the TerraSAR VV Polarization Image}

As microwave images have a granular appearance due to the speckle formed as a result of the coherent radiation used for radar systems, the reduction of the speckle is a very important step in further analysis. The analysis of the radar images must be based on the techniques that remove the speckle effects while considering the intrinsic texture of the image frame [8-10]. In this study, five different speckle suppression techniques such as local region, median, leesigma, frost, and gammamap filters [11] of $5 \times 5$ and 7 $\times 7$ sizes were compared in terms of delineation of urban features and texture information. After visual inspection of each image, it was found that the $5 \times 5$ gammamap filter created the best image in terms of delineation of different features as well as preserving content of texture information. In the output image, speckle noise was reduced with very low degradation of the textural information.

\section{Image Fusion}

In the present study, to update the database of 2000 up to the year of 2008, the Quickbird images of 2006 and TerraSAR image of 2008 had to be fused. Generally, image fusion is used for many purposes. Very often it is used to produce improved spatial resolution. The most common situation is represented by a pair of images where the first acquired by a multispectral sensor has a pixel size greater than the pixel size of the second image acquired by a panchromatic sensor. Combining these images, fusion produces a new multispectral image with a spatial resolution equal to the panchromatic one. In addition, image fusion introduces important distortions on the pixel spectra which in turn improve the information content of RS images [12]. Over the years, different fusion methods have been developed for improving spatial and spectral resolutions of RS data sets. The techniques most encountered in the literature are the intensity-hue-saturation (IHS) transform, the Brovey transform, the principal components analysis (PCA) method, the Gram-Schmidt method, the local mean matching method, the local mean and variance matching method, the least square fusion method, the wavelet-based fusion method, and the multiplicative and the Ehlers Fusion
[13-16]. Most fusion applications use modified approaches or combinations of these methods.

Now the fusion of optical and SAR data sets has been widely used for different applications. It has been found that the images acquired at optical and microwave ranges of electromagnetic spectrum provide unique information when they are integrated. As it is known, optical data contains information on the reflective and emissive characteristics of the Earth surface features, while the SAR data contains information on the surface roughness, texture, and dielectric properties of natural and man-made objects. It is evident that a combined use of the optical and SAR images will have a number of advantages because a specific feature which is not seen on the passive sensor image might be seen on the microwave image and vice versa because of the complementary information provided by the two sources [17-19]. Many authors have proposed and applied different techniques to combine optical and SAR images in order to enhance various features, and they all judged that the results from the fused images were better than the results obtained from the individual images [9, 14, 20-26].

In the case of the present study, for the urban areas, the radar image provides structural information about buildings and street alignment due to the double-bounce effect, while the optical images provide the information about the spectral and spatial variations of different urban features. Generally, image fusion can be performed at pixel, feature, and decision levels [21, 27]. In this study, data fusion has been performed at a pixel level, and the following rather common and more complex techniques were compared: (a) multiplicative method, (b) Brovey transform, (c) modified IHS (intensity, hue, and saturation), (d) principal component analysis (PCA), (e) Ehlers fusion, and (f) wavelet-based fusion. Each of these techniques is briefly discussed below.

5.1. Multiplicative Method. This is the most simple image fusion technique. It takes two digital images, for example, high-resolution panchromatic and low-resolution multispectral data and multiplies them pixel by pixel to get a new image [28].

5.2. Brovey Transform. This is a simple numerical method used to merge different digital data sets. The algorithm based on a Brovey transform uses a formula that normalises multispectral bands used for a red, green, and blue colour display and multiplies the result by high-resolution data to add the intensity or brightness component of the image [29]. For the Brovey transform, the bands of Quickbird data were considered as the multispectral bands, while the $\mathrm{HH}$-polarization of TerraSAR image was considered as the multiplying panchromatic band.

5.3. The Modified IHS. This method has a vast improvement over traditional IHS for fusing satellite imagery that differs noticeably in spectral response. It allows combining singleband panchromatic data with multispectral data, resulting in an output with both excellent detail and a realistic representation of original multispectral scene colors. The 
modified IHS method is designed to produce an output that approximates the spectral characteristics of the input multispectral bands while preserving the spatial integrity of the panchromatic data. The technique works by assessing the spectral overlap between each multispectral band and the high-resolution panchromatic band and weighting the merge based on these relative wavelengths [11].

5.4. PCA. The most common understanding of the PCA is that it is a data compression technique used to reduce the dimensionality of the multidimensional datasets [7]. It is also helpful for image encoding, enhancement, change detection, and multitemporal dimensionality [21]. PCA is a statistical technique that transforms a multivariate data set of intercorrelated variables into a set of new uncorrelated linear combinations of the original variables, thus generating a new set of orthogonal axes.

5.5. Ehlers Fusion. This is a fusion technique used for the spectral characteristics preservation of multitemporal and multisensor data sets. The fusion is based on an IHS transformation combined with filtering in the Fourier domain, and the IHS transform is used for optimal colour separation. As the spectral characteristics of the multispectral bands are preserved during the fusion process, there is no dependency on the selection or order of bands for the IHS transform $[14,30]$.

5.6. Wavelet-Based Fusion. The wavelet transform decomposes the signal based on elementary functions, that is the wavelets. By using this, an image is decomposed into a set of multiresolution images with wavelet coefficients. For each level, the coefficients contain spatial differences between two successive resolution levels. In general, a wavelet-based image fusion can be performed by either replacing some wavelet coefficients of the low-resolution image by the corresponding coefficients of the high-resolution image or by adding highresolution coefficients to the low-resolution data [31]. In this study, the first approach which is based on biorthogonal transforms has been applied.

In order to obtain good colour images that can illustrate spectral and spatial variations of the building and other classes on the selected optical and SAR images, all the fused images have been visually inspected and compared. In the case of the multiplicative method, the fused image demonstrated the worst result compared to all other combinations, while in the case of the Brovey transform, the combination of the Quickbird and TerraSAR created an image with some noise. The spectral appearance of the Brovey transformed image was a bit similar to the original Quickbird image, but on this image, the edges of the buildings were too much influenced by the speckle and textural information of the SAR image. In the case of the modified IHS method, the fused image demonstrated a better result compared to the combinations obtained by the multiplicative method and Brovey transform. However, a thorough inspection of the image had revealed that it still contained some noise of the radar image.
TABle 1: Principal component coefficients from Quickbird and TerraSAR images.

\begin{tabular}{lcccc}
\hline & PC1 & PC2 & PC3 & PC4 \\
\hline Quickbird PAN & 0.58 & 0.59 & 0.56 & 0.02 \\
Quickbird B3 & 0.01 & 0.01 & 0.02 & -0.99 \\
Quickbird B4 & -0.73 & 0.08 & 0.67 & 0.01 \\
TerraSAR VV & -0.35 & 0.80 & -0.48 & 0.00 \\
Eigenvalue & 11049.7 & 1914.2 & 259.8 & 28.8 \\
Variance (\%) & 83.37 & 14.44 & 1.96 & 0.23 \\
\hline
\end{tabular}

In the present study, the PCA has been performed using all available optical and SAR bands, and the results are shown in Table 1.

As can be seen from Table 1, PC1 contains $83.37 \%$ of the overall variance, whereas PC2 has $14.44 \%$ of the total variance. Thus, the first two PCs contain $97.81 \%$ of the overall variance. As seen, panchromatic band of Quickbird has high loadings in PC1, PC2, and PC3. Also, infrared band of Quickbird has the highest negative loading in PC1 and the highest loading in PC3. VV polarisation of TerraSAR data has the highest loading in PC2 and negative loading in PC3. Unlike the other bands, red band of Quickbird has no influence on the main PCs, but has a very high negative loading in the PC4. The inspection of the last PC that contained only $0.23 \%$ of the overall variance indicated that it contained noise from the total data set. Visual inspection of the image obtained by the PCA had shown that the image could clearly illustrate the individual buildings than any other images. However, the image contained too much colour variations of other classes, and it was very difficult to use for the interpretation.

In the case of the Ehlers fusion, the integrated image looked very similar to the original Quickbird image and demonstrated a better result compared to most other combinations. However, this image had a bit blurred appearance due to speckle noise of the SAR image which makes the image less relevant for the final interpretation. In the case of the wavelet-based fusion, the fused image demonstrated the best result compared to all other combinations. Although the image had a bit similar spectral appearance as the image obtained by the Ehlers fusion, it did not contain speckle. On this image, the buildings were very well separated from other classes both spatially and spectrally. Moreover, it could be seen that some textural information has been added for differentiation between the building and other classes. Therefore, the image obtained by the wavelet-based fusion has been used for the final analysis. Figure 2 shows the comparison of the images obtained by different fusion methods.

\section{Database Development and Land Use Change Analysis}

Initially, a digital topographic map of the study area of scale 1:5000 represented in a raster format has been georeferenced to a Gauss-Kruger map projection using 9 


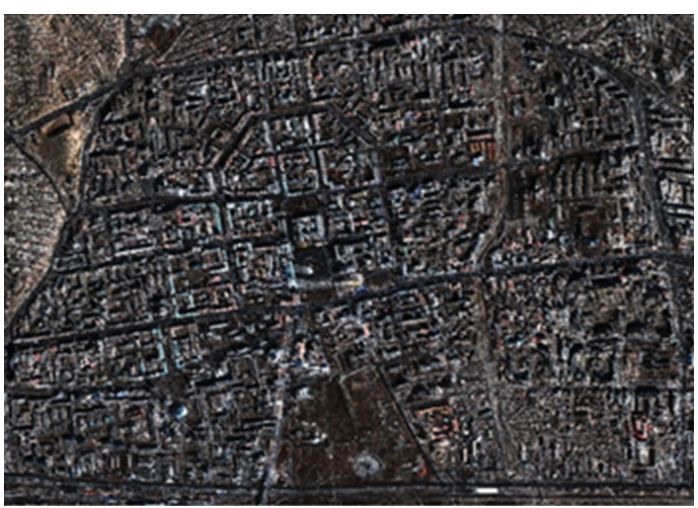

(a)

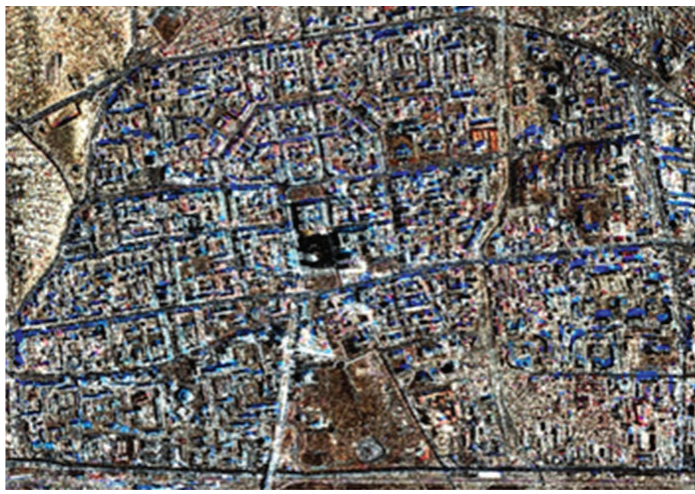

(b)

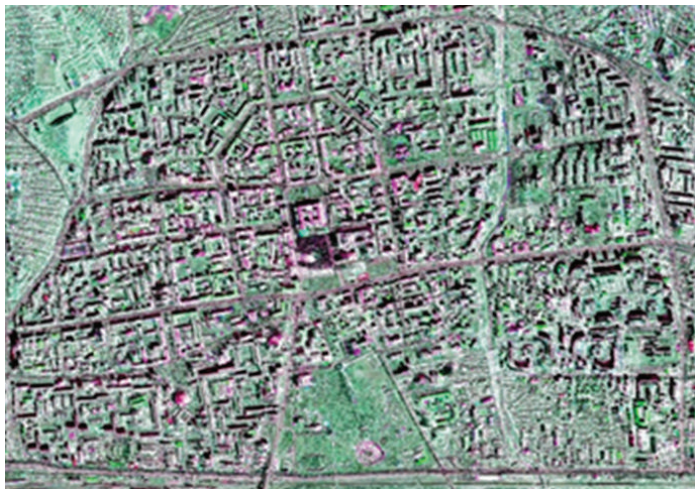

(c)

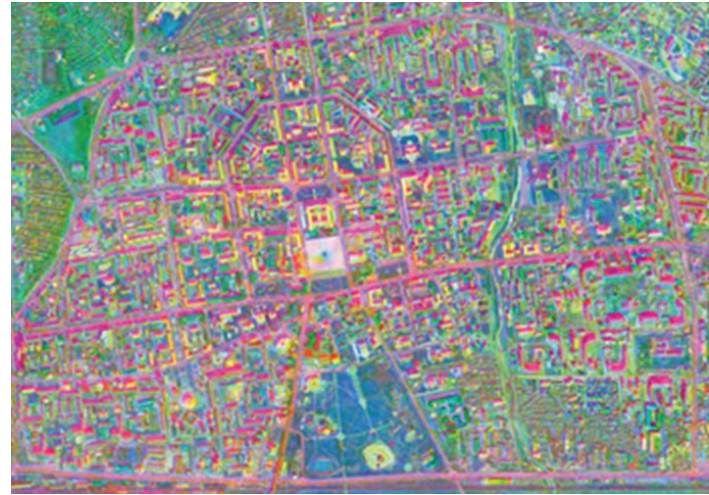

(d)

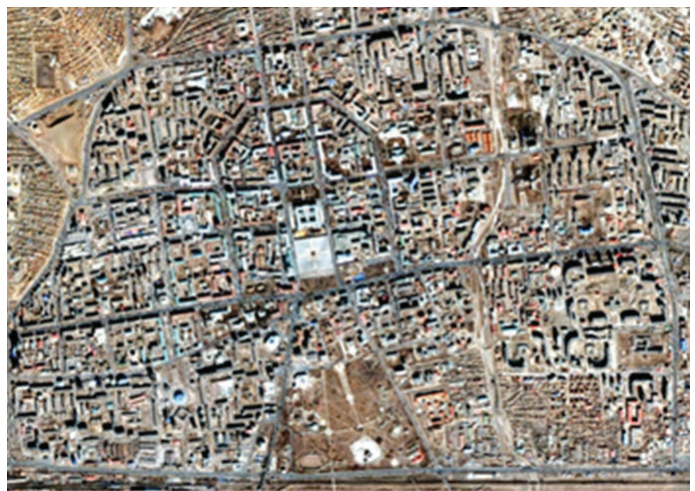

(e)

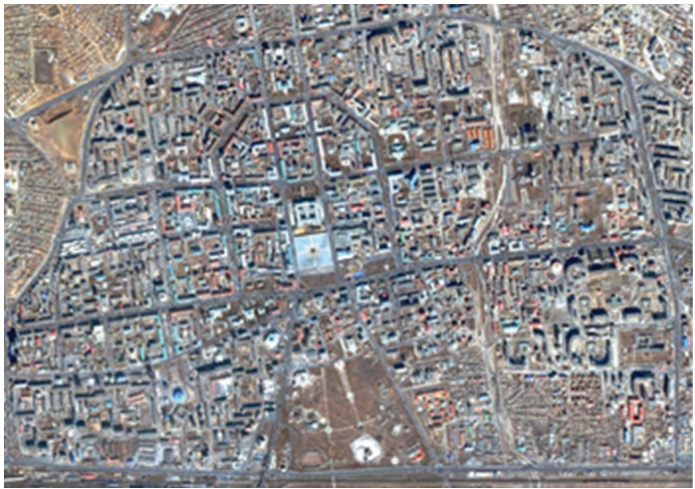

(f)

FIGURE 2: Comparison of the fused images: (a) multiplicative method, (b) Brovey transform, (c) modified IHS, (d) PC image (red = PC1, green $=$ PC2, and blue $=$ PC3), (e) Ehlers fusion, and (f) wavelet-based fusion.

GCPs. For the transformation, a linear transformation and nearest-neighbour resampling approach were applied, and the related RMS error was 0.26 pixel. In order to acquire primary digital data, the buildings were digitized from the georeferenced topographic map of 2000 using ArcGIS system. The digitized buildings illustrated on top of the topographic map are shown in Figure 3. Then, for each building entity, the attributes such as address, built year, use, condition, and storey number were entered. Moreover, the area of every building was calculated and stored as a new attribute within the database.
In order to analyse the changes occurred in between 2000 and 2008, it was necessary to update the created from the topographic map, database. For this purpose, the image obtained by the wavelet-based fusion has been used. For the thorough registration of the GIS and RS data sets, the coordinates of the fused image were transformed to the coordinates of the digitized map using 12 ground GCPs. For the transformation, a second-order transformation and nearest-neighbour resampling approach were applied, and the related RMS error was 0.82 pixel. Then, the digitized map was overlain on top of the georeferenced fused image thus 


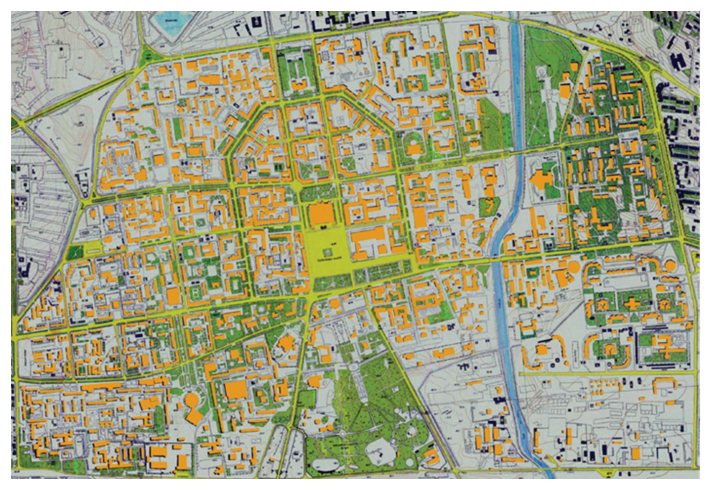

Digitized buildings overlain on top of the topographic map

Figure 3: A digitized map of Ikh Toiruu, overlain on top of a topographic map of 2000.

TABLE 2: Total number of buildings and their occupying areas (1930-2008).

\begin{tabular}{cccc}
\hline No. & Time period & Number of buildings & Area (sq.m) \\
\hline$(1)$ & $1930-1940$ & 14 & 19139.92 \\
$(2)$ & $1940-1950$ & 19 & 27394.71 \\
$(3)$ & $1950-1960$ & 104 & 140520.63 \\
$(4)$ & $1960-1970$ & 166 & 167826.43 \\
$(5)$ & $1970-1980$ & 150 & 185715.96 \\
$(6)$ & $1980-1990$ & 118 & 149354.96 \\
$(7)$ & $1990-2000$ & 276 & 268406.59 \\
$(8)$ & $2000-2008$ & 509 & 372762.25 \\
\hline & Total: & 1356 & 1331121.45 \\
\hline
\end{tabular}

highlighting the buildings appeared after 2000. After this, on the georeferenced image, the buildings were screen digitized and updated the previously created database. After that for all new building entities, the attributes such as address, built year, use, condition, and storey number were entered. Like before, the area of each new building entity was calculated and updated the database. The updated map is shown in Figure 4. To analyse the changes occurred from 1930 to 2008 with a 10-year interval, queries have been made using "Select by attributes" function of ArcGIS. After selecting the buildings occurring in each of the selected time interval, the related number of buildings and their occupying areas have been calculated (Table 2). Figure 5 shows colour differences of the buildings occurring in different time periods.

As could be seen from Table 2 and Figure 5, in the study area, there were built very few buildings until 1950 (i.e., 14 buildings having $19139.92 \mathrm{~m}^{2}$ in $1930-1940$ and 19 buildings having $27394.71 \mathrm{~m}^{2}$ in $1940-1950$ ). This is due to a fact that after the World War II, the country's economy was poor, and there were very few development activities in Mongolia. However, commencing from 1950, due to the country's economy increase, a new development started, and within a decade, many new buildings had been built (i.e., 104 buildings having $140520.63 \mathrm{~m}^{2}$ ). From 1960 to 1980 , Mongolia was at the highest of its economic

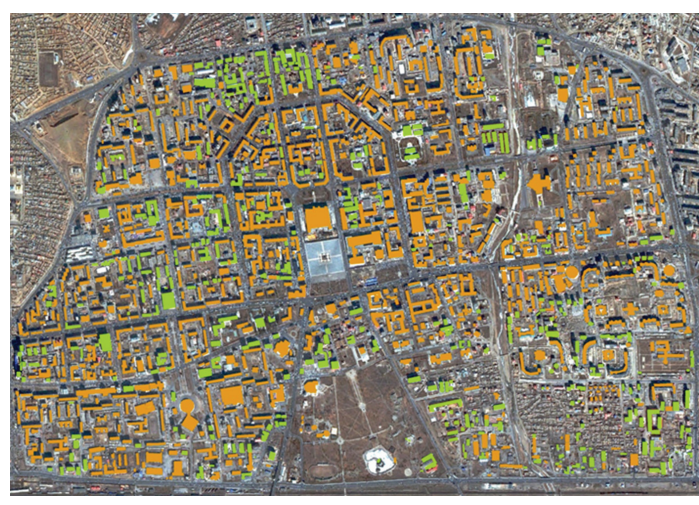

Buildings built before 2000

Buildings built after 2000

FIgURE 4: The updated map of Ikh toiruu, 2008.

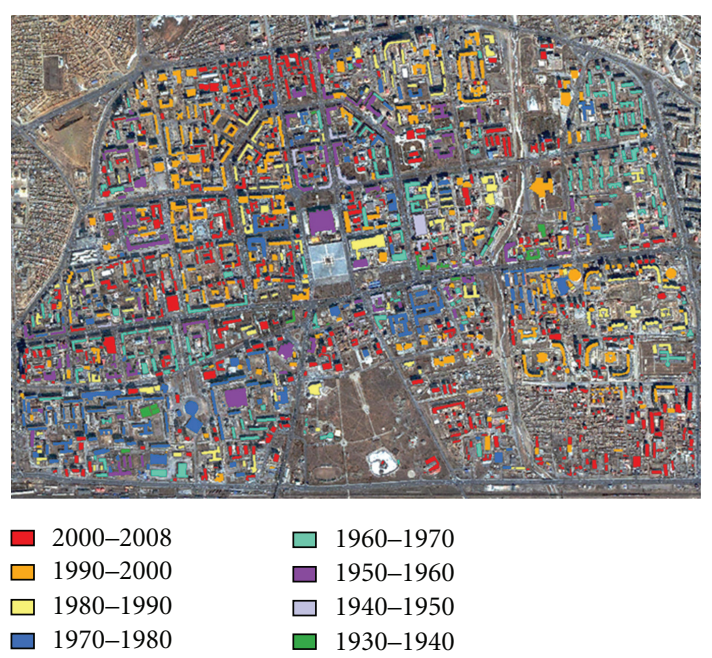

Figure 5: Distribution of buildings built from 1930 to 2008 (with a 10 year interval).

development. During this period, a significant number of residential, commercial, and governmental buildings had been built within the city's central zone (i.e., 166 buildings having $167826.43 \mathrm{~m}^{2}$ in $1960-1970$ and 150 buildings having $185715.96 \mathrm{~m}^{2}$ in 1970-1980). As can be seen, there were built only 118 buildings having $149354.96 \mathrm{~m}^{2}$ in $1980-1990$. This is related with the decline of the Mongolian economy by the end of 1980s which was common for all communist countries at that time. In 1990, Mongolia entered market economy, and the interests of people to own land parcels and build houses, have greatly increased. Although throughout the 1990s, the country's economy still was in a difficult condition, there were built many new buildings in Ikh toiruu area (i.e., 276 buildings having $268406.59 \mathrm{~m}^{2}$ ). From the new century, Mongolian economy started to improve, and people wanted to have a piece of land in areas having good infrastructure and build something on it. Due to this reason, there was a rapid increase of the buildings in the city's central zone. As seen from Table 2, there were built 509 buildings having $372762.25 \mathrm{~m}^{2}$ in $2000-2008$, in this area. 


\section{Conclusions}

The idea of the research was to analyze urban land use changes occurred in central part of Ulaanbaatar city from 1930 to 2008 with a 10-year interval using GIS and very highresolution optical and SAR data sets. As data sources, $1: 5000$ scale topographic map, panchromatic and multispectral Quickbird images, and TerraSAR data were used. The primary urban land use database was developed using the topographic map of the study area and historical data about buildings. To extract updated land use information from the RS images, Quickbird and TerraSAR images were fused. For the data fusion, multiplicative method, Brovey transform, modified IHS, PCA, Ehlers fusion, and wavelet-based fusion were compared. Of these methods, the image obtained by the wavelet-based fusion gave a superior image in terms of the differentiation between the buildings and other urban classes. To extract reliable land use information from the fused image, a visual interpretation was applied. As could be seen from the analysis, in the study area, there were built 571 buildings having 689952.6 sq.m until 1990. However, since Mongolia entered the market economy in 1990, the interests of people to own land parcels and build houses have greatly increased, and there were built 792 buildings having 645892.9 sq.m. Overall, the study demonstrated that within the last few decades the central part of Ulaanbaatar was urbanized very rapidly and became very dense.

\section{Acknowledgments}

A part of this research was conducted under the sponsorship of Asia Research Center, National University of Mongolia. The authors are grateful to Professor J. L. van Genderen from the ITC for providing free TerraSAR data this study.

\section{References}

[1] D. Amarsaikhan, H. H. Blotevogel, M. Ganzorig, and T. H. Moon, "Applications of remote sensing and geographic information systems for urban land-cover change studies in Mongolia," Geocarto International, vol. 24, no. 4, pp. 257-271, 2009.

[2] C. L. Redman and N. S. Jones, "The environmental, social, and health dimensions of urban expansion," Produced for a Population-Environment Research Network cyberseminar, 2004, http://www.populationenvironmentresearch.org/ papers/Urban_Expansion_background_paper.pdf.

[3] D. Amarsaikhan, M. Ganzorig, and T. H. Moon, "Application of multitemporal RS and GIS data for urban change studies," in Proceedings of the Korean GIS Conference, pp. 190-215, Busan, South Korea, 2005.

[4] A. Gruen, "Reality-based generation of virtual environments for digital earth," International Journal of Digital Earth, vol. 1, no. 1, pp. 88-106, 2008.

[5] A. Kumar, A. C. Pandev, N. Hoda, and A. T. Jeyaseelan, "Evaluation of urban sprawl pattern in the tribal-dominated cities of Jharkhand state, India," International Journal of Remote Sensing, vol. 32, no. 22, pp. 7651-7675, 2011.

[6] D. Amarsaikhan, B. Chinbat, M. Ganzorig, V. Battsengel, and R. Gantuya, "Applications of RS and GIS for urban land use change study in Ulaanbaatar city, Mongolia," Journal of Geography and Regional Planning, vol. 4, no. 8, pp. 471-481, 2011.

[7] J. A. Richards and S. Xia, Remote Sensing Digital Image Analysis-An Introduction, Springer, Berlin, Germany, 3rd edition, 1999.

[8] F. T. Ulaby, F. Kouyate, B. Brisco, and T. H. L. Williams, "Textural information in SAR images," IEEE Transactions on Geoscience and Remote Sensing, vol. 24, no. 2, pp. 235-245, 1986.

[9] D. Amarsaikhan and T. Douglas, "Data fusion and multisource image classification," International Journal of Remote Sensing, vol. 25, no. 17, pp. 3529-3539, 2004.

[10] M. Serkan, N. Musaoglu, H. Kirkici, and C. Ormeci, "Edge and fine detail preservation in SAR images through speckle reduction with an adaptive mean filter," International Journal of Remote Sensing, vol. 29, no. 23, pp. 6727-6738, 2008.

[11] ERDAS, Field Guide, ERDAS, Atlanta, Ga, USA, 5th edition, 1999.

[12] S. Teggi, R. Cecchi, and F. Serafini, "TM and IRS-1C-PAN data fusion using multiresolution decomposition methods based on the 'a tròs' algorithm," International Journal of Remote Sensing, vol. 24, no. 6, pp. 1287-1301, 2003.

[13] V. Karathanassi, P. Kolokousis, and S. Ioannidou, "A comparison study on fusion methods using evaluation indicators," International Journal of Remote Sensing, vol. 28, no. 10, pp. 2309-2341, 2007.

[14] M. Ehlers, S. Klonus, and P. J. Åstrand, "Quality Assessment for Multi-sensor Multi-date Image Fusion," in Proceedings of ISPRS Congresses, Beijing, China, July 2008.

[15] J. Soria-Ruiz, Y. Fernandez-Ordońez, and I. H. Woodhouse, "Land-cover classification using radar and optical images: a case study in Central Mexico," International Journal of Remote Sensing, vol. 31, no. 12, pp. 3291-3305, 2010.

[16] F. Chen, Z. Guan, X. Yang, and W. Cui, "A novel remote sensing image fusion method based on independent component analysis," International Journal of Remote Sensing, vol. 32, no. 10, pp. 2745-2763, 2011.

[17] D. Amarsaikhan, M. Ganzorig, G. Batbayar, D. Narangerel, and S. H. Tumentsetseg, "An integrated approach of optical and SAR images for forest change study," Asian Journal of Geoinformatics, vol. 3, pp. 27-33, 2004.

[18] D. Amarsaikhan, M. Ganzorig, P. Ache, and H. Blotevogel, "The integrated use of optical and InSAR data for urban landcover mapping," International Journal of Remote Sensing, vol. 28, no. 6, pp. 1161-1171, 2007.

[19] J. Zhang, "Multi-source remote sensing data fusion: status and trends," International Journal of Image and Data Fusion, vol. 1, no. 1, pp. 5-24, 2010.

[20] Y. Wang, B. N. Koopmans, and C. Pohl, "The 1995 flood in the Netherlands monitored from space-a multi-sensor approach," International Journal of Remote Sensing, vol. 16, no. 15, pp. 2735-2739, 1995.

[21] C. Pohl and J. L. Van Genderen, "Multisensor image fusion in remote sensing: concepts, methods and applications," International Journal of Remote Sensing, vol. 19, no. 5, pp. 823854, 1998.

[22] E. Ricchetti, "Visible-infrared and radar imagery fusion for geological application: a new approach using DEM and sunillumination model," International Journal of Remote Sensing, vol. 22, no. 11, pp. 2219-2230, 2001.

[23] N. D. Herold and B. N. Haack, "Fusion of radar and optical data for land cover mapping," Geocarto International, vol. 17, no. 2, pp. 21-30, 2002. 
[24] T. Westra, K. C. Mertens, and R. R. De Wulf, "ENVISAT ASAR wide swath and SPOT-VEGETATION image fusion for wetland mapping: Evaluation of different wavelet-based methods," Geocarto International, vol. 20, no. 2, pp. 21-31, 2005.

[25] N. M. Saadi and K. Watanabe, "Assessing image processing techniques for geological mapping: a case study in Eljufra, Libya," Geocarto International, vol. 24, no. 3, pp. 241-253, 2009.

[26] M. Ehlersa, S. Klonusa, P. J. Åstrandb, and P. Rossoa, "Multisensor image fusion for pansharpening in remote sensing," International Journal of Image and Data Fusion, vol. 1, no. 1, pp. 25-45, 2010.

[27] M. A. Abidi and R. C. Gonzalez, Data Fusion in Robotics and Machine Intelligence, Academic Press, New York, NY, USA, 1992.

[28] M. Seetha, B. L. Malleswari, I. V. MuraliKrishna, and B. L. Deekshatulu, "Image fusion-a performance assessment," Journal of Geomatics, vol. 1, no. 1, pp. 33-39, 2007.

[29] J. Vrabel, "Multispectral imagery band sharpening study," Photogrammetric Engineering and Remote Sensing, vol. 62, no. 9, pp. 1075-1083, 1996.

[30] M. Ehlers, "Spectral characteristics preserving image fusion based on Fourier domain filtering," in Remote Sensing for Environmental Monitoring, GIS Applications, and Geology IV, vol. 5574 of Proceedings of SPIE, pp. 1-13, September 2004.

[31] G. Pajares and J. M. de la Cruz, "A wavelet-based image fusion tutorial,” Pattern Recognition, vol. 37, no. 9, pp. 1855-1872, 2004. 

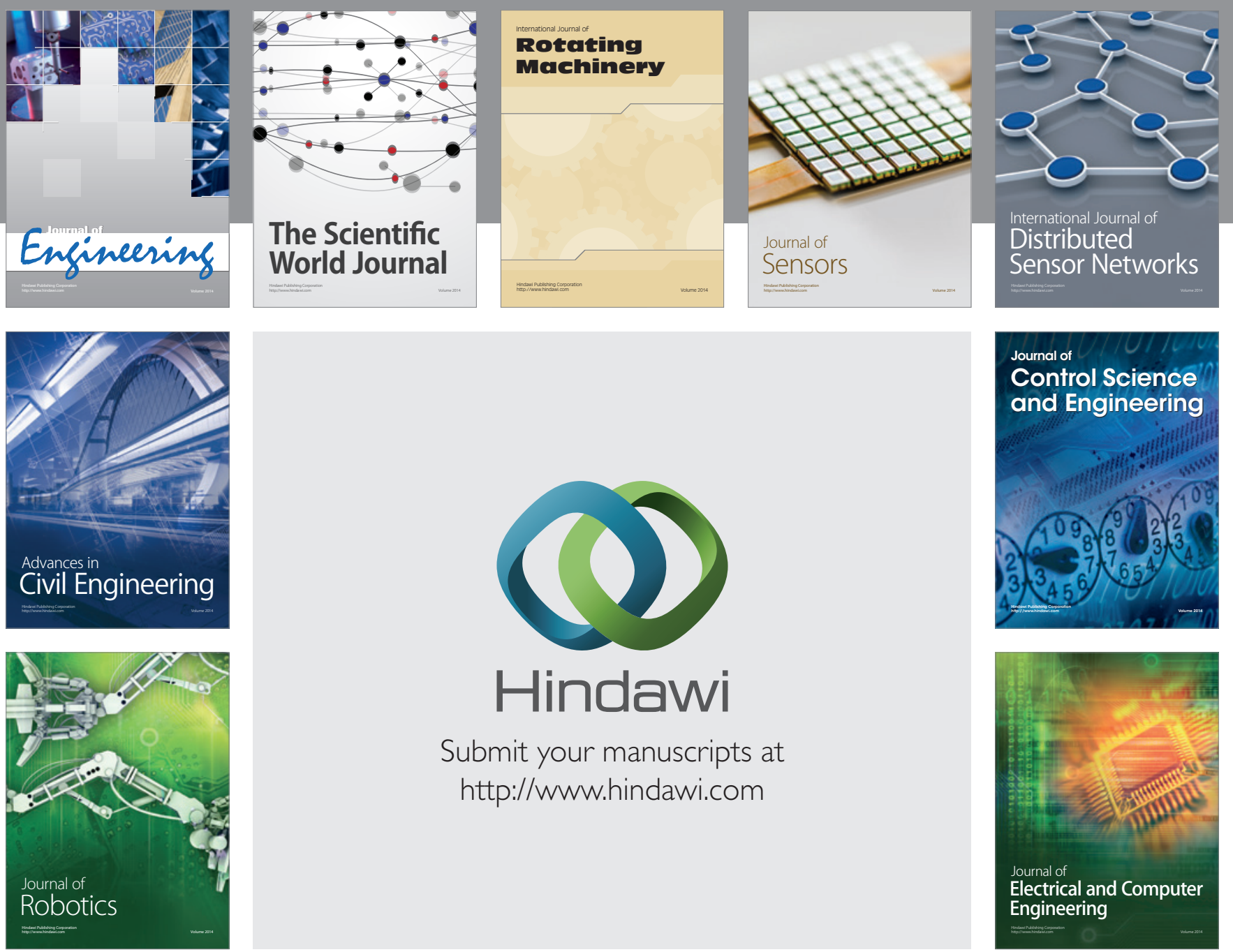

Submit your manuscripts at

http://www.hindawi.com
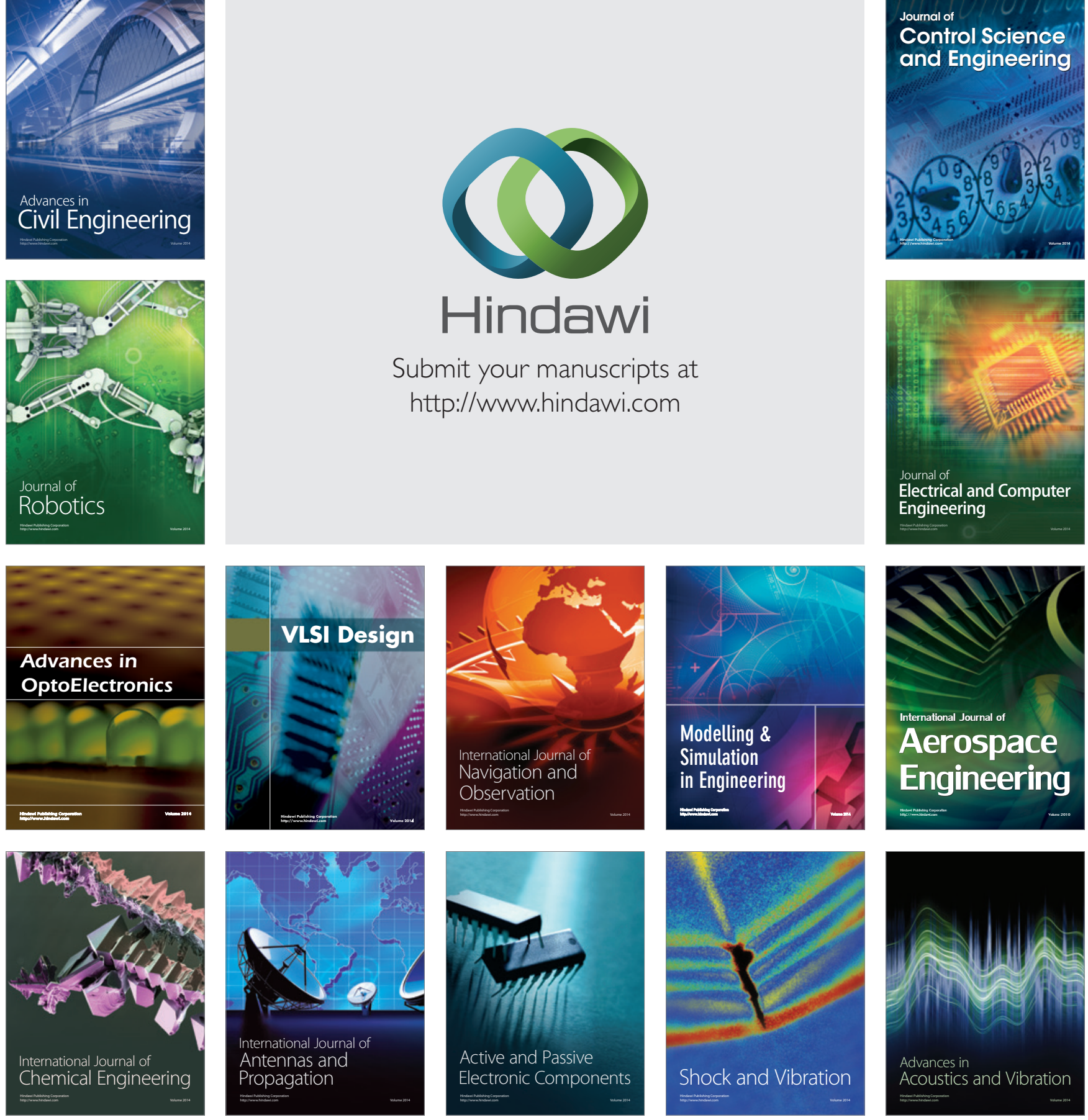